\title{
Effects of a 12-month physical activity counselling intervention on glycaemic control and on the status of cardiovascular risk factors in people with Type 2 diabetes
}

\author{
A. Kirk ${ }^{1,3} \cdot$ N. Mutrie ${ }^{1} \cdot$ P. MacIntyre ${ }^{1}$ M. Fisher $^{2}$ \\ ${ }^{1}$ Centre for Exercise Science and Medicine, University of Glasgow, Scotland \\ 2 Diabetes Centre, Glasgow Royal Infirmary, Scotland \\ ${ }^{3}$ Institute of Sport and Exercise, University of Dundee, Dundee, Scotland
}

\section{Abstract}

Aims/hypothesis. The aim of this study was to investigate the effectiveness of physical activity counselling in promoting physical activity in people with Type 2 diabetes and to evaluate resultant physiological and biochemical effects.

Methods. A total of 70 inactive people with Type 2 diabetes were given standard exercise information and randomised to receive physical activity counselling ( $n=35$, experimental) or not ( $n=35$, control). Physical activity consultations were delivered at baseline and after 6 months, with follow-up phone calls after 1, 3, 6 and 9 months. Changes from baseline after 6 and 12 months were assessed for physical activity (7-day recall and accelerometer), for physiological characteristics (body mass index and blood pressure) and for biochemical variables $\left(\mathrm{HbA}_{1} \mathrm{c}\right.$, lipid profile, fibrinogen, tissue plasminogen activator and microalbuminuria).

Results. Significant differences between groups were recorded for physical activity after 6 and 12 months $(p<0.01)$. The experimental group had increased levels of physical activity from baseline to 6 months $(p<0.01)$, with no decrease from 6 to 12 months $(p>0.05)$. In the control group, accelerometer counts per week decreased from baseline to 12 months $(p=0.03)$. Between-group differences $(p<0.05)$ were recorded for the change in $\mathrm{HbA}_{1} \mathrm{c}$ (experimental: $0.26 \%$ decrease; control: $0.15 \%$ increase), for systolic blood pressure (experimental: $7.7 \mathrm{~mm} \mathrm{Hg}$ decrease; control: $5.6 \mathrm{~mm} \mathrm{Hg}$ increase) and for fibrinogen (experimental: $0.28 \mathrm{mmol} / \mathrm{l}$ decrease; control: $1.43 \mathrm{mmol} / \mathrm{l}$ increase) from baseline to 6 months, and for total cholesterol (experimental: $0.33 \mathrm{mmol} / \mathrm{l}$ decrease; control: $0.04 \mathrm{mmol} / \mathrm{l}$ increase) from baseline to 12 months $(p<0.05)$. No significant differences were recorded in other measured variables.

Conclusions/interpretations. Physical activity counselling was effective in promoting physical activity in people with Type 2 diabetes. The counselling improved glycaemic control as well as the status of cardiovascular risk factors in these patients.

Keywords Cardiovascular risk factors - Glycaemic control - Physical activity - Physical activity counselling · Type 2 diabetes
Received: 8 December 2003 / Accepted: 21 February 2004 Published online: 5 May 2004

C) Springer-Verlag 2004

\section{A. Kirk (๘)}

Institute of Sport and Exercise, University of Dundee, DD1 4HN Dundee, Scotland

E-mail: a.kirk@dundee.ac.uk

Tel.: +44-1382-348853, Fax: +44-1382-344121

Abbreviations: CSA, Computer Science and Applications . t-PA, tissue plasminogen activator antigen - UKPDS, United Kingdom Prospective Diabetes Study

\section{Introduction}

Extensive evidence demonstrates the benefits of physical activity for the management of Type 2 diabetes. A meta-analysis [1] reviewed the effect of exercise on glycaemic control in people with Type 2 diabetes and demonstrated an overall mean difference in $\mathrm{HbA}_{1} \mathrm{c}$ of $0.66 \%$ between exercise and control groups. This clinically significant improvement, if maintained, has the potential to substantially reduce the development of diabetic complications [2]. Regular physical activity also has the potential to improve the status of several 
Table 1. Physical activity interventions for people with Type 2 diabetes

\begin{tabular}{|c|c|c|c|c|}
\hline Author & $\begin{array}{l}\text { Study } \\
\text { type }\end{array}$ & Intervention & Population & $\begin{array}{l}\text { Outcomes \& } \\
\text { follow-up }\end{array}$ \\
\hline
\end{tabular}

Lightenberg et al. 1997 RCT Supervised aerobic 58 elderly men Physical activity, [8]

Mourier et al. 1997

[9]

Raz et al. 1994

[10]

Lehmann et al. 1995

[11]

Lehmann et al. 2001

[12]

Samsara 1997

[13]

Vanninen et al. 1992

[14]

Uusitupa 1996

[15]

Hanefeld et al. 1991

[16]
RCT Supervised aerobic exercise

RCT Supervised aerobic exercise programme

RCT Supervised exercise programme

29 people with Type 2 diabetes

16 people with

Case Supervised exercise control programme

RCT

Structured exercise programme, including support for 6 months (provided monthly) compared with usual care

RCT Diet \& exercise inte
vention. Oral and written instruction for physical activity

RCT Multifactorial intervention with 6 visits compared to standard care. Oral and written instruction for physical activity

RCT Multifactorial intervention. Supervised structured exercise programme and active living encouraged Type 2 diabetes

40 people with Type 2 diabetes

Type 2 diabetes

26 sedentary people with Type 2 diabetes

Type 2 diabetes

78 people with Type 2 diabetes

86 people with Type 2 diabetes
24 people with

$\mathrm{VO}_{2(\max )}$, body composition, $\mathrm{HbA}_{1} \mathrm{c}$. 2 months follow-up full lipid profile, body composition. 6 months follow-up

Attendance at exercise class, $\mathrm{VO}_{2(\max )}$, BMI, $\mathrm{HbA}_{1} \mathrm{c}$, total cholesterol, HDL cholesterol, LDL cholesterol, triglycerides. 3 months follow-up

Physical activity, blood pressure, body composition, lipid profile, $\mathrm{HbA}_{1}$ c. 3 months follow-up

Physical activity (monitored by daily records). Full lipid profile, $\mathrm{HbA}_{1} \mathrm{c}$, body composition. 3 months follow-up

Anthropometric measures, lipid profile, $\mathrm{HbA}_{1} \mathrm{c}$, physical activity. 6 and 12 months follow-up $\mathrm{VO}_{2(\max )}$, physical activity questionnaire. 12 months follow-up

Physical activity and $\mathrm{VO}_{2(\max )} .12$ months follow-up

1139 people with Physical activity diet-controlled questionnaire. Type 2 diabetes 5 years follow-up
Significant increase in $\mathrm{VO}_{2(\max )}$ and physical activity in experimental group. No change in controls. Significant between-group difference of change in total cholesterol

Significant improvements in all outcomes in experimental group. No changes in controls Significant improvement in $\mathrm{VO}_{2(\max )}, \mathrm{HbA}_{1} \mathrm{c}$ and triglycerides in experimental group. No changes in controls

Significant improvements in physical activity, blood pressure, body composition and lipid profile in experimental group. No change in $\mathrm{HbA}_{1} \mathrm{c}$. No changes in controls

Significant improvements in physical activity, body

composition, HDL

cholesterol and total cholesterol in experimental group. No changes in controls

, No change in physical activity behaviour in experimental group. Significant difference between groups in $\mathrm{HbA}_{1} \mathrm{c}$ after 6 months, not maintained after 12 months. No change in any other outcomes

No change in physical activity or $\mathrm{VO}_{2(\max )}$ in either group

No change in physical activity in either group

Significantly higher physical experimental group. No change in controls. Only 20-30\% attended exercise sessions after 2 years 
Table 1 (continued)

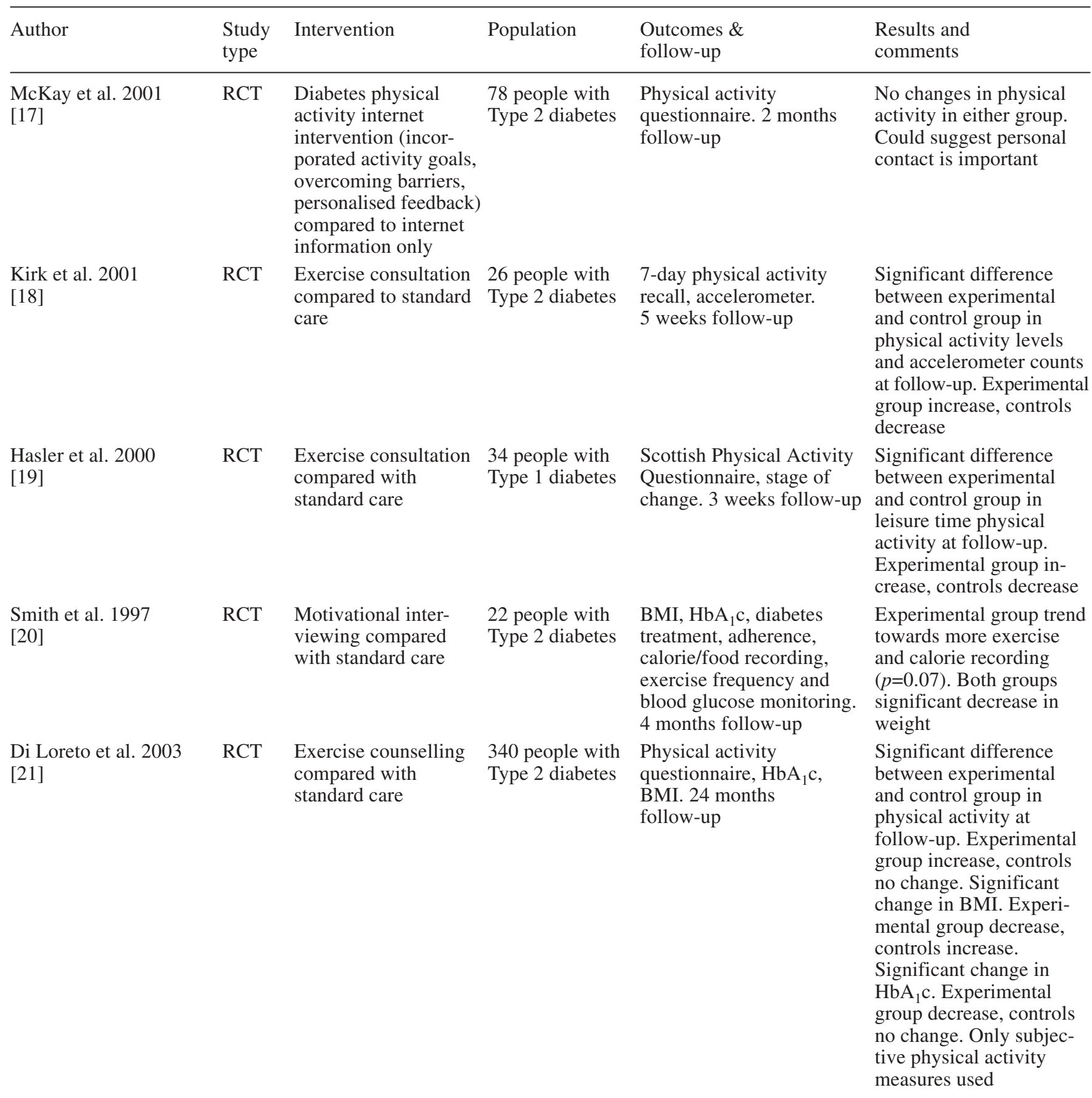

$\mathrm{RCT}$, randomised controlled trial; $\mathrm{VO}_{2(\max )}$, maximal oxygen uptake

cardiovascular risk factors such as body composition, blood pressure, blood lipids, cardiorespiratory fitness and fibrinolytic functioning, as well as quality of life and perceived well-being [3]. Furthermore, recent intervention trials have shown that diet plus exercise can reduce or delay the progression of impaired glucose tolerance to Type 2 diabetes by $58 \%[4,5]$.

Up to $80 \%$ of people with Type 2 diabetes do not do enough physical activity to benefit their health, and in comparison with the general population, people with diabetes experience a higher frequency of relapse to sedentary behaviour [6]. Promotion of physical activity in current diabetes care is inadequate. People with Type 2 diabetes report receiving less support, less education and less encouragement to undertake physical activity than reported for any other area of diabetes management [7].

Table 1 details previous research involving physical activity interventions for people with Type 2 diabetes. The majority of the research has involved struc- 
tured exercise programmes. These programmes are effective for increasing physical activity, and for improving glycaemic control and the status of cardiovascular risk factors in people with Type 2 diabetes over the short term (up to 6 months). However, such programmes rarely have a theoretical basis, and often target a motivated group of healthy people. In addition, they are expensive to develop and maintain, and they achieve poor long-term adherence. A large prospective study reported that only $25 \%$ of their Type 2 diabetic subjects were still engaged with their supervised exercise programmes after 2 years [16]. Required are alternative interventions that are effective in stimulating long-term changes in physical activity behaviour and that can be realistically implemented into current diabetes care.

A recent systematic review [22] provided strong evidence for the effectiveness of physical activity counselling in promoting behavioural change in the intermediate and long term. These interventions are particularly effective for attracting sedentary people who want to increase their physical activity [23]. The characteristics of physical activity counselling make it an attractive intervention for promoting physical activity in people with Type 2 diabetes. A person's physical condition and level of motivation can be considered and used to tailor the physical activity programme. An educational component can be included, involving discussion of the metabolic effects of exercise and ways to avoid unnecessary risks. Physical activity counselling could potentially be conducted by any member of a multidisciplinary diabetes team, following appropriate training, and could realistically be included into current diabetes care with minimal expense.

While there is no universal consensus on the most effective theoretical basis for physical activity promotion, a large amount of research supports the use of the transtheoretical model of behavioural change [24]. This model proposes that when changing behaviour, individuals move through stages: (i) pre-contemplation (no intention to change); (ii) contemplation (considering change); (iii) preparation (making small changes); (iv) action (actively engaging in new behaviour); and (v) maintenance (sustaining the change over time). The model includes three mediators that have been shown to be important for behavioural change: (i) self-efficacy (confidence in ability to change); (ii) decisional balance (pros and cons of change); and (iii) processes of change (strategies and techniques used to change, e.g. social support).

Using this evidence, an existing individualised physical activity counselling intervention [25] based on the transtheoretical model [24] was adapted to promote physical activity in people with Type 2 diabetes. Two randomised controlled pilot studies have shown this intervention to be effective in promoting shortterm behavioural change (for 1 month) in people with Type 1 [19] and Type 2 [18] diabetes.
The aim of this project was to evaluate the longerterm effectiveness (after 6 and 12 months) of physical activity counselling in promoting and maintaining physical activity in people with Type 2 diabetes, and to evaluate the resultant changes in physiological, biochemical and quality of life variables. Preliminary results (after 6 months) from this study have been previously reported [26]. The changes in behaviour (physical activity levels, stages and processes of behavioural change) will be reported separately. Whilst reporting the changes in physical activity levels after 6 and 12 months, the main focus of this paper is the resultant changes in physiological and biochemical variables.

\section{Subjects and methods}

\section{Participants}

A total of 223 people with Type 2 diabetes, identified from diabetes out-patient clinics, were invited to participate in this study. From this group, 70 people actually participated. Reasons for not participating were lack of time, person already participating in a clinical trial, or person not meeting inclusion criteria. There were no significant differences between the sampled group and the recruited group in terms of sex, age and BMI $(p>0.05)$. The study group consisted of 35 men and 35 women with a mean age of 57.6 \pm 7.9 years, and a mean BMI of 34.6 \pm 6.8 . Diabetes mellitus was defined according to established criteria [27] and was controlled by diet, oral hypoglycaemic agents or insulin. Participants were in either the contemplation or the preparation stage of exercise behavioural change, i.e. they were not following current physical activity guidelines, but they did intend to become more active [24]. Participants with concurrent medical conditions preventing exercise were excluded. The ethics committee of Argyll and Clyde Health Board, Scotland, approved this study, and written informed consent was obtained from all participants.

\section{Procedures}

The progress of the participants through the trial is illustrated in Figure 1. For baseline assessments, personal and medical details were recorded, and body weight and height were measured to determine BMI. Participants were asked to perform a peak exercise test. This test was used to screen participants for silent ischaemic heart disease prior to randomisation and to assess cardiorespiratory fitness (data previously reported [26]). Participants were given a Computer Science and Applications (CSA) physical activity monitor [28] to be worn for 7 days, and a sample bottle to provide a 24-hour urine sample. Blood pressure was measured and a fasting blood sample was taken to measure full lipid profile, glycaemic control and concentrations of fibrinogen and tissue plasmingen activator antigen (t-PA). The 7-day physical activity recall interview [29] was completed. All participants then received standard exercise information, in the form of a leaflet approved by Diabetes UK, and were randomly assigned to the experimental or control (standard care) group.

Random assignment was carried out on an individual basis in blocks of 20, using consecutively numbered sealed envelopes. Only experimental participants returned for a third visit. During this visit, participants received a physical activity 


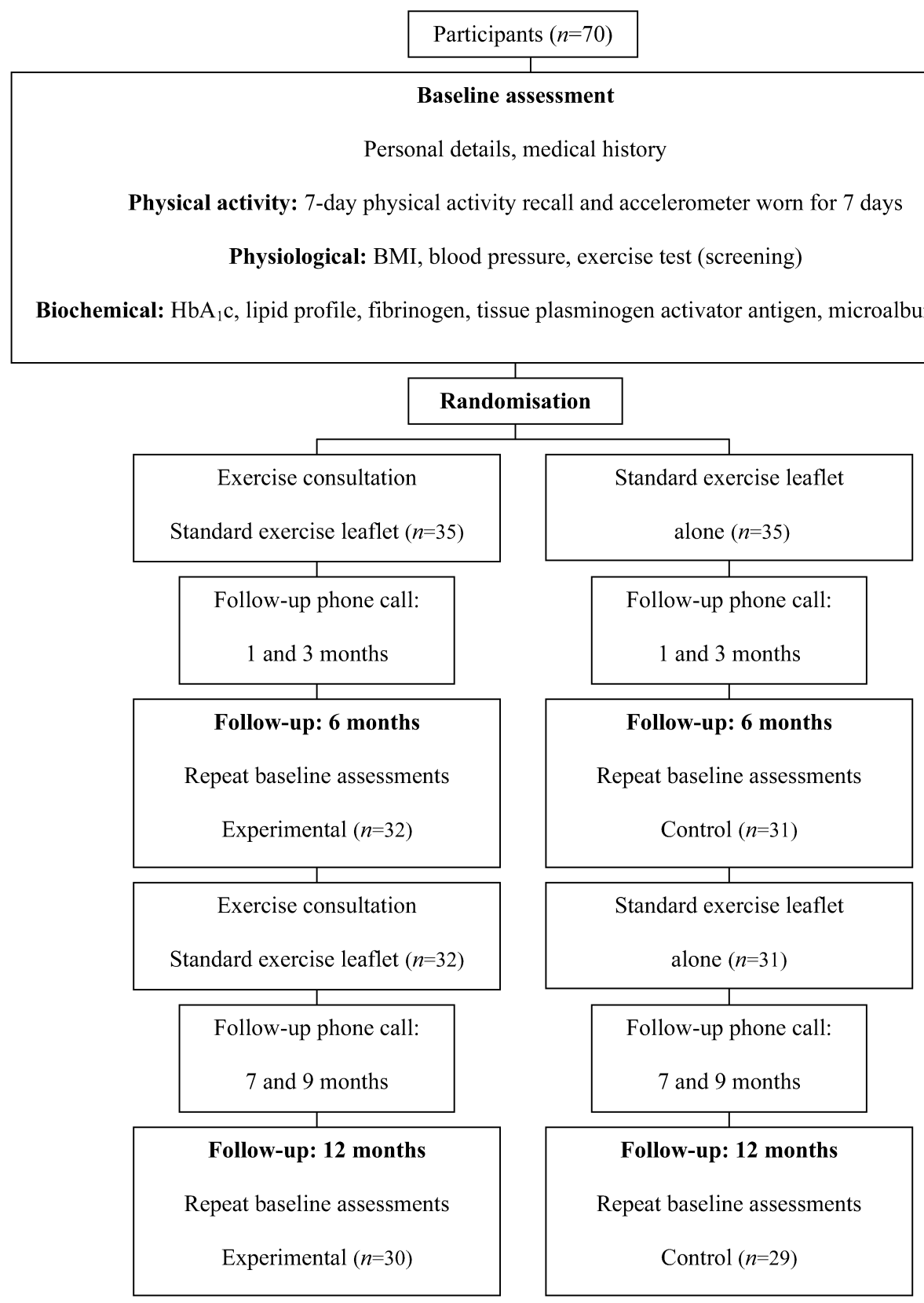

Fig. 1. Progression of participants through the trial

consultation. After 6 and 12 months, all outcome measures and procedures carried out at baseline were repeated, with the exception of physical activity counselling which was not repeated after 12 months.

\section{Intervention}

Physical activity counselling. Physical activity counselling was conducted following previously published guidelines [25], and involved a one-to-one discussion with a trained research assistant lasting approximately 30 minutes. During the intervention, stage of behavioural change was confirmed, and stage-tailored strategies and techniques known to influence the mediators of change were used to encourage physical activity. At baseline, the strategies and techniques used were similar for all, as each participant was in either the contemplation or the preparation stage. The strategies and techniques included investigating current physical activity, investigating the benefits, barriers and costs of becoming more active, finding suitable activities, providing social support, setting goals, and preventing relapse to sedentary behaviour. For participants in the contemplation stage, more emphasis was placed on increasing motivation to become more active. For participants in the preparation stage, more emphasis was placed on developing a suitable physical activity plan.

After the initial consultation, a number of participants had progressed to a higher stage of behavioural change. Physical activity consultations given after 6 months focused more on preventing relapse to sedentary behaviour and on improving long-term maintenance of a physically active lifestyle. Relapse prevention strategies involved identifying situations that may have a negative impact on behavioural change, such as a busy work schedule or holidays, and developing ways to prevent relapse during these high-risk situations, e.g. discussion of time management and cues to get back into an activity plan after 
holidays. A number of strategies used in baseline consultations were also incorporated into the consultation after 6 months. The benefits, costs and barriers encountered by the participants were discussed, and current physical activity status was reviewed. Individualised time-phased physical activity goals (for 1, 3 and 6 months) were again negotiated.

The aim of the consultation was to encourage patients to accumulate 30 minutes of moderate physical activity on most days of the week, as recommended by the American College of Sports Medicine and by Centers for Disease Control and Prevention [30]. If the participant was already undertaking this level of activity, they were encouraged to progress towards the American College of Sports Medicine 1990 guidelines of three 20 -min sessions of continuous, moderate to hard exercise per week to improve fitness [31].

Follow-up support phone calls were given 1 and 3 months after each consultation. During these phone calls a general discussion was conducted of previously set activity goals, experienced benefits, barriers and costs of becoming more active, and relapse prevention. To maintain equal contact time between groups, control participants also received a phone call. For the control group these phone calls involved discussions about topics unrelated to exercise.

Standard exercise leaflet. A leaflet entitled 'Exercise and your Diabetes', approved by Diabetes UK, was used as standard exercise information to provide a minimal service to the control group. The leaflet covered the following topics: why a person with diabetes should exercise; recommended amount of exercise; how to get started; and how exercise can affect diabetes. The leaflet was given to both experimental and control participants. The content of the leaflet was not explained to any participant.

\section{Outcome measures}

Physical activity outcomes. The 7-day physical activity recall [29] was used as a subjective measure of physical activity and involves a structured interview following a standard set of questions. Time spent per week asleep, as well as time spent undertaking light, moderate, hard, very hard, and strengthening and flexibility activities was recorded.

The CSA uniaxial accelerometer (Computer Science and Applications, Shalimar, Fla., USA) was used to provide an objective assessment of physical activity measured by bodily acceleration [28]. Monitors were secured by a strap to the participant's right ankle and worn for 7 days during all waking hours except bathing. The CSA monitor records the magnitude of accelerations during body movement at a rate of ten samples per second. These samples were totalled to produce activity counts at 1-minute intervals. During return visits, monitors were downloaded and a total weekly activity count was calculated.

Physiological outcomes. BMI was calculated as weight $(\mathrm{kg}) /$ height $(\mathrm{m})^{2}$. Blood pressure was measured from the left arm in the seated position using an automatic blood pressure monitor (Omron 705CP; Omron Healthcare, Japan). Three blood pressure measurements were taken and the average value recorded.

Biochemical outcomes. A blood sample was taken with minimal venous occlusion after an overnight fast of 12 hours, and analysed for $\mathrm{HbA}_{1} \mathrm{c}$, total cholesterol, HDL cholesterol, LDL cholesterol, triglycerides, fibrinogen and t-PA. $\mathrm{HbA}_{1} \mathrm{c}$ was measured by latex-enhanced turbidimetric immunoassay [32] (non-diabetic range: $4.5-6.0 \%$ ). Total cholesterol, triglycerides and HDL cholesterol were measured directly using enzymatic methods [33], and LDL cholesterol was calculated using the Friedewald formula [34]. Fibrinogen was measured by the Clauss method [35], and t-PA was measured by enzyme immunoassay [36].

The number of participants in each group on potentially confounding medication (for hypoglycaemia, hypotensive or cardiovascular therapy) was recorded at each time point and was analysed using chi square tests to determine any significant differences between groups. No data were collected on adherence to medication or dietary therapy.

\section{Statistical analysis}

Estimation of sample size. Statistical power was calculated using the difference in the mean change in physical activity counts per week between the experimental and control groups after 30 days, obtained from a pilot study [18]. There was a $90 \%$ power of detecting a true $23 \%$ difference in counts per week (baseline mean: 1813893; mean difference: 409780; SD: 444779 ) with a minimum of 25 participants in each group, given a significance level of 5\%. To allow for drop-out, 35 participants were recruited in each group. In view of the time constraints for this study, statistical power was not adequate to analyse sex-dependent effects.

Analysis. Data were analysed using Minitab (version 13.30). A total of 35 experimental and 35 control data sets were analysed for the 7-day recall, CSA accelerometer, BMI and blood pressure. There were some missing data for biochemical outcomes (see Table 1) due to problems obtaining blood samples. Repeated measures (time), two-way (time $\times$ group) ANOVA models were used to analyse the effect of the intervention on measured outcomes. Where appropriate, covariate adjustments for effects of sex and BMI were applied. Significant main effects were followed up with Bonferroni multiple comparisons for repeated measures. For the repeated measures analyses, tests were conducted for homogeneity of covariance using a Mauchly sphericity test. If data failed this test $(p<0.05)$, a Greenhouse Geisser modification was used. Anderson-Darling tests were performed to test for normality. Variables with nonnormal distributions (7-day physical activity recall) were transformed using the square root before analysis. Data analysis was conducted on an intention-to-treat basis and with missing data removed. For all variables the same values of significance were obtained, suggesting that no significant response bias was caused by study drop-out. Categorical data are reported as a proportion and were analysed using chi square or Fisher's exact test. We considered a $p$ value of less than 0.05 to be statistically significant. On the 7-day recall, only a small number of participants took part in hard, very hard, and strengthening and flexibility activities. These categories were combined with moderate activity and analysed as total activity.

\section{Results}

No between-group differences were found on any measured variable at baseline.

Physical activity outcomes. Analyses of the 7-day recall revealed significant main effects of time $\left(F_{2.68}=6.2 ; p=0.003\right)$, group $\left(F_{1.68}=16.7 ; p<0.001\right)$ and the interaction of time and group $\left(F_{2.68}=7.5 ; p=0.008\right)$. 


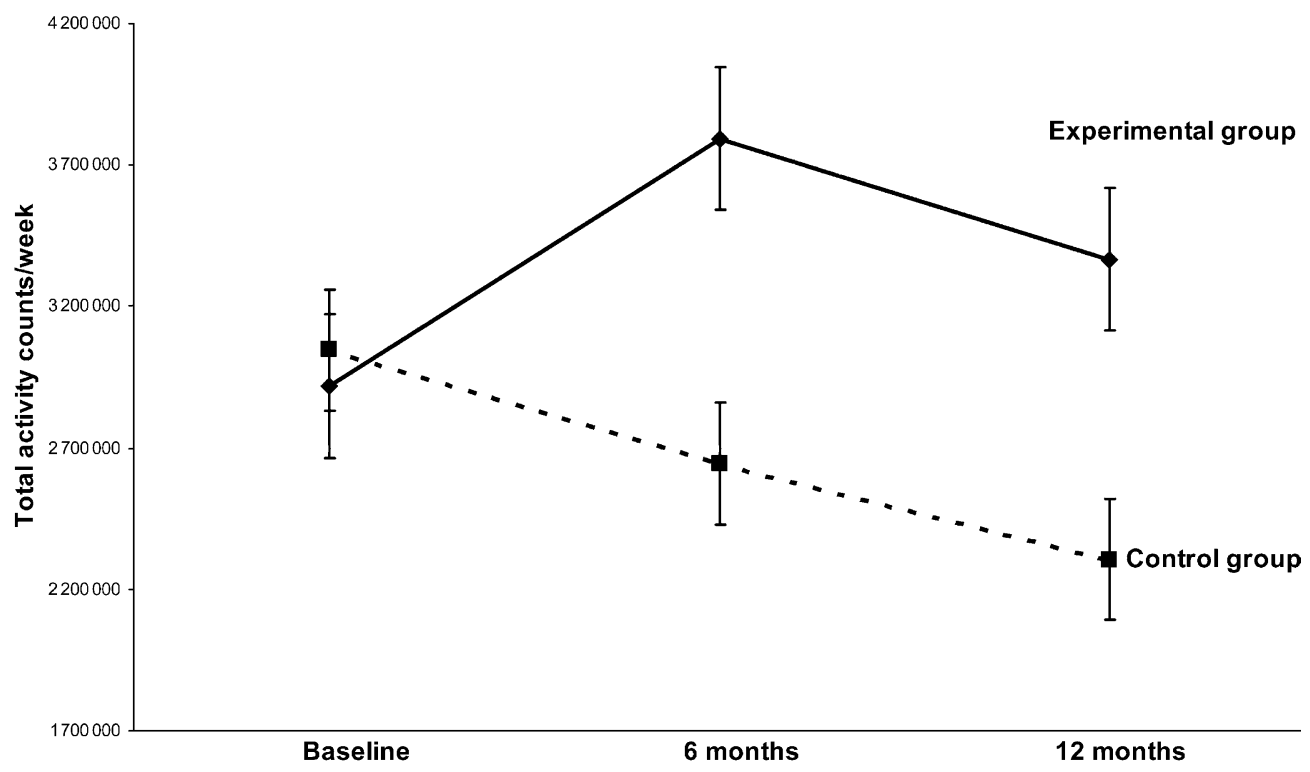

Fig. 2. Total activity counts/week at baseline, after 6 months and after 12 months according to group. Data are means with standard error bars

Bonferroni multiple comparisons illustrated that the experimental group showed a significant increase in total minutes of activity from baseline to 6 months (mean difference: $70.3 \mathrm{~min}$; 95\% CI: 22.2 to $145.3 \mathrm{~min}$ ) and 12 months (mean difference: $55.2 \mathrm{~min}$; $95 \%$ CI: 14.1 to $123.2 \mathrm{~min}$ ), with no significant decrease from 6 to 12 months (mean difference: $-10.2 \mathrm{~min}, 95 \% \mathrm{CI}$ : -21.4 to $7.4 \mathrm{~min}$ ). The control group recorded no significant changes from baseline after either 6 months (mean difference: $-20.2 \mathrm{~min}$; 95\% CI: -44.0 to $0.3 \mathrm{~min}$ ) or 12 months (mean difference: $-13.7 \mathrm{~min}$; $95 \% \mathrm{CI}:-35.7$ to $1.5 \mathrm{~min}$ ). Significant between-group differences were recorded after 6 months (mean difference: $97.0 \mathrm{~min} ; 95 \% \mathrm{CI}:-161.7$ to $-38.6 \mathrm{~min}$ ) and 12 months (mean difference: $67.9 \mathrm{~min}$; $95 \% \mathrm{CI}$ : -164.6 to $-30.9 \mathrm{~min}$ ) with the experimental group reporting more total activity than controls after both 6 and 12 months.

Figure 2 shows the total activity counts with SE recorded by the CSA accelerometer at baseline, after 6 months and after 12 months. For the CSA accelerometer, repeated measures ANOVA identified a significant main effect of group $\left(F_{1.68}=34.9 ; p<0.01\right)$ and the interaction of group and time $\left(F_{2.68}=10.0 ; p<0.01\right)$. Bonferroni multiple comparisons for repeated measures illustrated that the experimental group significantly increased total activity counts per week from baseline to 6 months (mean difference: 805576 counts; 95\% CI: 188427 to 1422725 counts/week), with no significant decrease from 6 to 12 months (mean difference: -388944 counts; 95\% CI: -1040090 to 262202 counts/week). The change from baseline to 12 months did not reach significance (mean difference: 416632 counts; $95 \%$ CI: -217743 to 1051007 counts/week). The control group recorded no significant change in total activity counts per week from baseline to 6 months (mean difference: -333343 counts; 95\% CI: -932741 to 266054 counts/week) or 6 to 12 months (mean difference: -335717 counts; 95\% CI: -966683 to 295248 counts/week), although a significant decrease was recorded from baseline to 12 months (mean difference: -669061 counts; 95\% CI: -1292285 to -45837 counts/week). Significant between-group differences were recorded after 6 months (mean difference: -1139129 counts; 95\% CI: -1786768 to -491490 counts/week) and 12 months (mean difference: -1085903 counts; 95\% CI: -1771560 to -400245 counts/week), with the experimental group recording higher total activity counts per week than controls after both 6 and 12 months.

There was a significant correlation $(p<0.01)$ between total activity from the 7-day recall and total activity counts from the CSA accelerometer for the experimental $(r=0.43)$ and control $(r=0.37)$ group at baseline.

Physiological outcome measures. Analysis of BMI demonstrated no significant main effect of time $\left(F_{2.68}=1.6 ; p=0.2\right)$, group $\left(F_{1.68}=0.05 ; p=0.8\right)$ or the interaction of time and group $\left(F_{2.68}=1.5 ; p=0.2\right)$. There were no significant differences between the experimental and control groups for the change in BMI from baseline to 6 months (mean difference: $-0.7 ; 95 \% \mathrm{CI}$ : -1.7 to 0.3 ) or 12 months (mean difference: -0.9 ; 95\% CI: -1.9 to 0.1 ).

Figure 3 illustrates the change in systolic and diastolic blood pressure by group from baseline after 6 and 12 months. Analysis of changes in blood pressure revealed no significant main effect of time $\left(F_{2.68}=0.3\right.$; $p=0.7)$, group $\left(F_{1.68}=0.9 ; p=0.3\right)$ or the interaction of time and group $\left(F_{2.68}=2.7 ; p=0.08\right)$. The difference between the experimental and control groups for the change in systolic and diastolic blood pressure from 

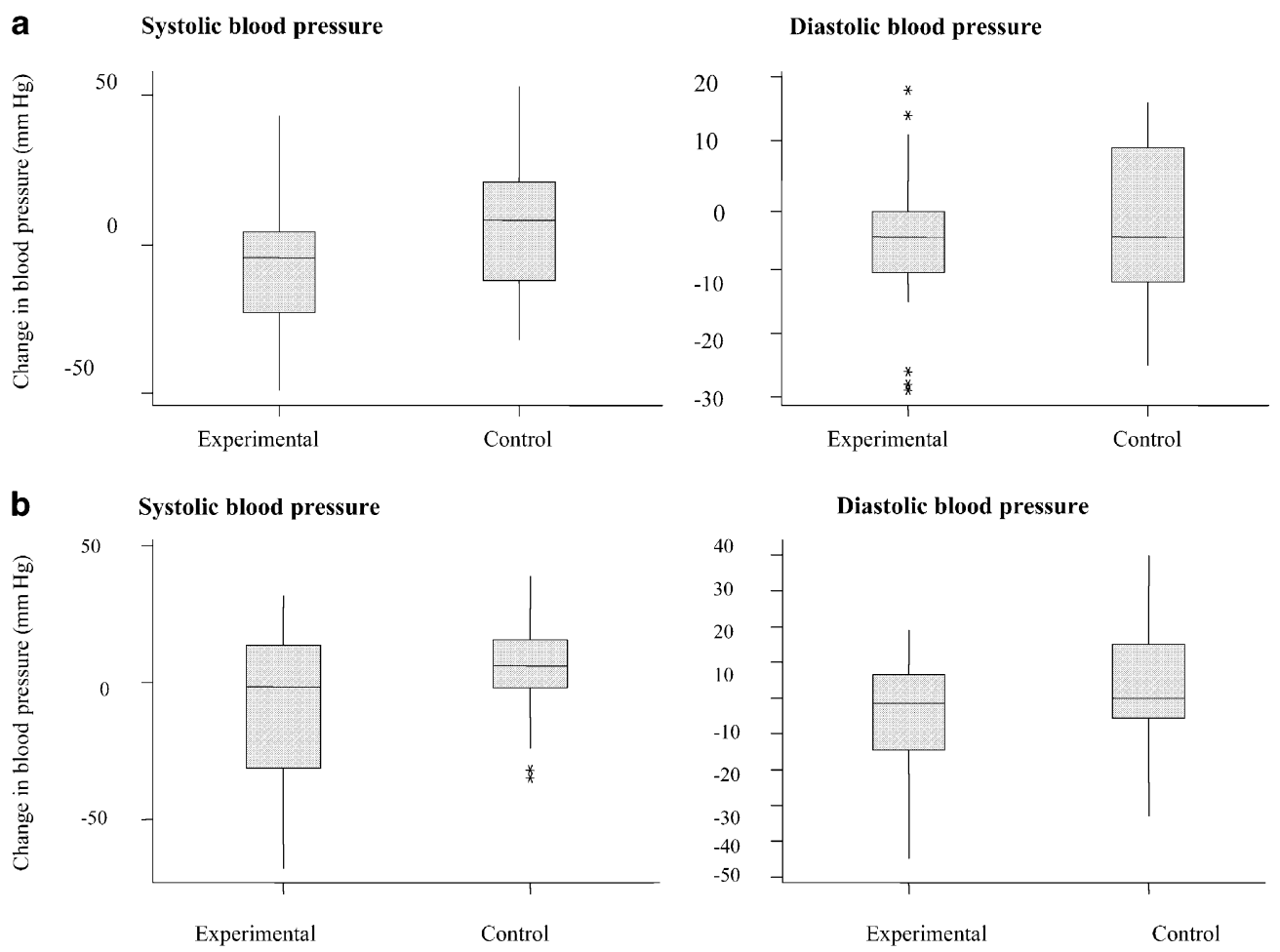

Fig. 3. Change in systolic and diastolic blood pressure from baseline after (a) 6 months and (b) 12 months according to group. Data are means; *outliers

vealed no significant between-group differences in the number of participants on hypotensive medication at baseline $\left(\chi^{2}=0.06, d f=2 ; p=0.9\right)$, after 6 months $\left(\chi^{2}=0.2, d f=2 ; p=0.9\right)$ or after 12 months $\left(\chi^{2}=0.7\right.$, $d f=2 ; p=0.7)$.

baseline to 6 and 12 months revealed a significant between-group difference for the change in systolic blood pressure from baseline to 6 months (95\% CI: -24.7 to $-2.0 \mathrm{~mm} \mathrm{Hg}$ ). The experimental group recorded a small decrease $(6.3 \mathrm{~mm} \mathrm{Hg})$ and the control group recorded a small increase $(5.0 \mathrm{~mm} \mathrm{Hg})$. No significant differences were recorded between groups for the change in diastolic blood pressure from baseline to 6 months (mean difference: $-4.05 \mathrm{~mm} \mathrm{Hg}$; 95\% CI: -9.8 to 1.7 ) and 12 months (mean difference: $-6.91 \mathrm{~mm} \mathrm{Hg}$; $95 \% \mathrm{CI}:-15.2$ to $1.4 \mathrm{~mm} \mathrm{Hg}$ ) or the change in systolic blood pressure from baseline to 12 months (mean difference: $-12.32 \mathrm{~mm} \mathrm{Hg}$; $95 \% \mathrm{CI}$ : -24.7 to $0.1 \mathrm{~mm} \mathrm{Hg}$ ). Blood pressure was also categorised at baseline, after 6 months and after 12 months according to whether or not it was in line with the current guidelines (below 140/80) [37]. At baseline, 11 out of 35 experimental participants and 13 out of 35 control participants had blood pressure results in line with these guidelines. After 6 months, 16 out of 30 experimental participants and 8 out of 31 control participants had results in line with the guidelines, and after 12 months this had changed to 11 out of 28 experimental participants and 3 out of 29 control participants. Chi square analyses revealed a greater number of experimental participants were in line with the guidelines after 6 months $\left(\chi^{2}=4.8, d f=1 ; p=0.03\right)$ and 12 months $\left(\chi^{2}=6.4, d f=1 ; p=0.01\right)$. Chi square re-

Biochemical outcomes. The changes in biochemical variables are illustrated in Table 2 . There was no significant main effect of time $\left(F_{2.63}=0.2 ; p=0.9\right)$ or the interaction of time and group $\left(F_{2.63}=2.1 ; p=0.1\right)$ recorded for the changes in $\mathrm{HbA}_{1} \mathrm{c}$, but there was a significant effect of group $\left(F_{1.63}=6.6 ; p=0.01\right)$. Follow-up Bonferroni multiple comparisons for repeated measures showed that the mean $\mathrm{HbA}_{1} \mathrm{c}$ was significantly different between the groups after 6 months (95\% CI: 0.49 to $1.9 \%$ ) and 12 months (95\% CI: 0.22 to $1.7 \%$ ). There was a significant difference between the experimental and control groups for the change in $\mathrm{HbA}_{1} \mathrm{c}$ from baseline to 6 months (95\% CI: -1.23 to $-0.07 \%$ ) but not from baseline to 12 months $(95 \% \mathrm{CI}$ : -1.32 to $0.5 \%$ ). Chi square analyses of diabetes therapy revealed no significant between-group differences at baseline $\left(\chi^{2}=3.7, d f=3 ; p=0.3\right)$, after 6 months $\left(\chi^{2}=3.6, d f=3 ; p=0.03\right)$ or after 12 months $\left(\chi^{2}=1.8\right.$, $d f=3 ; p=0.6)$.

There was no significant main effect of time, group or the interaction of time and group for the changes in total cholesterol, HDL cholesterol, LDL cholesterol, triglycerides or t-PA. The differences between the experimental and control groups for the change in each variable from baseline to 6 months and baseline to 12 months were also analysed. The only significant difference recorded was in total cholesterol from baseline to 12 months ( $95 \% \mathrm{CI}:-0.73$ to $-0.04 \mathrm{mmol} / \mathrm{l}$ ). 
Table 2. Biochemical variables at baseline, after 6 months and after 12 months according to group

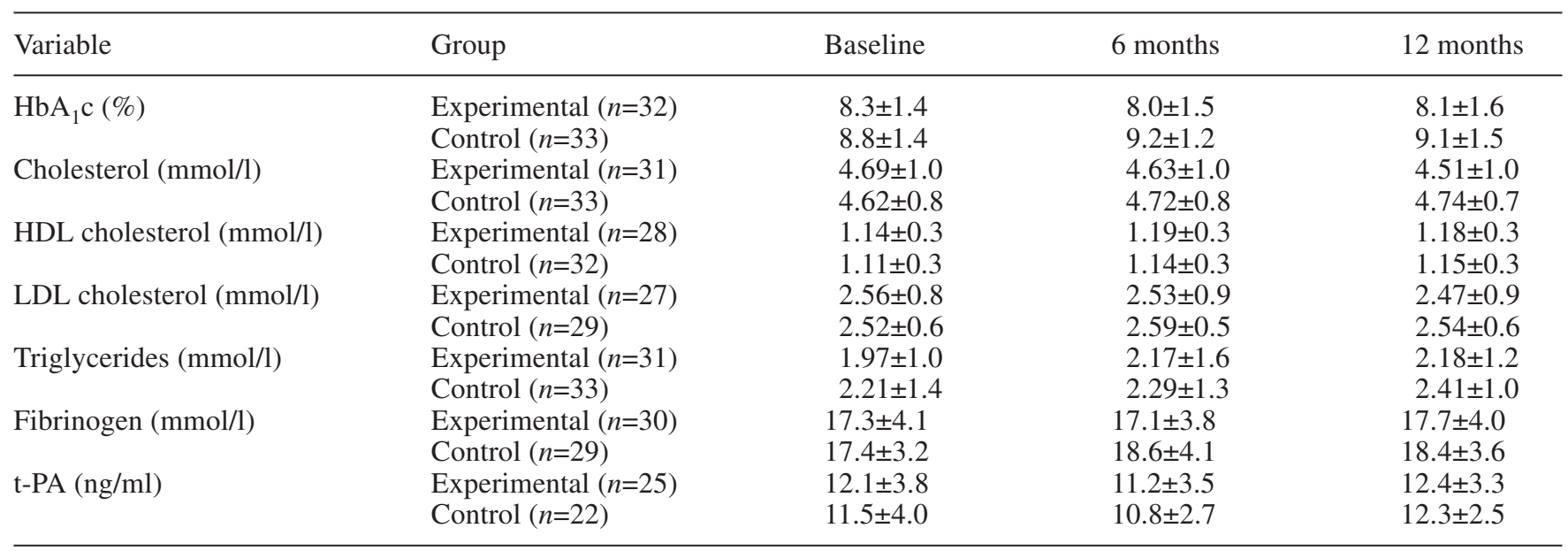

Data are means $\pm \mathrm{SD}$

Analysis of fibrinogen concentrations revealed a significant main effect of time $\left(F_{2.57}=3.7 ; p=0.3\right)$ and the interaction of time and group $\left(F_{2.57}=3.0 ; p=0.05\right)$, but no significant main effect of group $\left(F_{1.57}=0.69\right.$; $p=0.4$ ). Follow-up Bonferroni multiple comparisons for repeated measures showed that after 6 months the experimental and control groups were significantly different $(95 \% \mathrm{CI}: 0.18$ to $1.73 \mu \mathrm{mol} / \mathrm{l})$. The difference between the experimental and control groups for the change in fibrinogen concentrations was significant from baseline to 6 months $(95 \% \mathrm{CI}:-2.04$ to $-0.15 \mu \mathrm{mol} / \mathrm{l}$ ) but not from baseline to 12 months (95\% CI: -1.44 to $0.31 \mu \mathrm{mol} / \mathrm{l})$. Chi square analyses revealed no significant between-group differences for the number of people with evidence of microalbuminuria at baseline $\left(\chi^{2}=1.2, d f=1 ; p=0.3\right)$, after 6 months $\left(\chi^{2}=2.0, d f=1 ; p=0.2\right)$ or after 12 months $\left(\chi^{2}=1.2\right.$, $d f=1 ; p=0.3)$. Chi square analyses demonstrated no significant difference in the number of participants in each group on cardiovascular medication.

\section{Discussion}

Consistent changes in subjective and objective measures of physical activity have demonstrated that physical activity counselling was more effective in promoting and maintaining physical activity in people with Type 2 diabetes than a standard exercise leaflet.

A key finding of this study is that increases in physical activity from baseline to 6 months in the experimental group were generally maintained after 12 months. Although the change in accelerometer counts from baseline to 12 months did not reach significance, the difference between the experimental and control groups after 12 months is significant. A limited amount of research has evaluated the longer-term effects of physical activity interventions in people with Type 2 diabetes. A large prospective study re- ported that only $25 \%$ of people with Type 2 diabetes were still engaged with their supervised exercise programme 2 years later [16]. Two studies [14, 15] reported no significant change in physical activity 12 months after giving people with Type 2 diabetes written and oral instructions for exercise. The interventions used in these studies were not tailored to motivational or personal characteristics, and they did not have cognitive behavioural strategies or a theoretical basis. Effective physical activity promotion over 2 years in people with Type 2 diabetes has previously been demonstrated using a physical activity counselling intervention [21]. Similar to the intervention used in the present study, the physical activity counselling intervention was individualised, and it incorporated cognitive behavioural strategies. In contrast to this study, the intervention was not tailored to stage of behavioural change, it only used self-reported physical activity and it was delivered by physicians. In the current study, any member of the diabetes team with appropriate training could deliver the intervention. This could be more cost-effective than a physiciandelivered intervention.

No significant between- or within-group changes were recorded in BMI over the study period. These results contrast with those of a previous study [21] that reported a significant favourable change in BMI in people with Type 2 diabetes after a 2-year physical activity counselling intervention. A meta-analysis [1] of studies investigating the effect of exercise on BMI in people with Type 2 diabetes showed no significant greater change in BMI when exercise conditions were compared with control conditions. The majority of studies included in this meta-analysis had follow-up periods of between 2 and 6 months. The disparity in results could be a consequence of the different interventions or of the different durations of follow-up.

Participants receiving physical activity counselling experienced small favourable changes in blood pres- 
sure despite having no significant changes in antihypertensive medication. Two recent systematic reviews [38, 39] demonstrated that there is good evidence that regular, moderate physical activity reduces blood pressure in the general population. Only a small number of studies have investigated the effect of regular physical activity on blood pressure in people with Type 2 diabetes. Lehmann et al. [11] reported significant reductions in systolic $(138 \pm 16$ to $128 \pm$ $15 \mathrm{~mm} \mathrm{Hg})$ and diastolic $(88 \pm 10$ to $77 \pm 6 \mathrm{~mm} \mathrm{Hg})$ blood pressure after a 3 -month moderate-intensity aerobic exercise programme in people with Type 2 diabetes. These improvements are slightly higher than those recorded in this study and could be due to the shorter follow-up, the supervised nature of the physical activity intervention or the type of activity undertaken. In the United Kingdom Prospective Diabetes Study (UKPDS), tight blood pressure control reduced the development and progression of diabetic complications, and reduced risk of death related to diabetes [40].

Analyses of changes in $\mathrm{HbA}_{1} \mathrm{c}$ showed a significant between-group difference after 6 and 12 months, with the experimental group experiencing an improvement in glycaemic control, and the control group experiencing a deterioration. These changes occurred despite no significant between-group changes in diabetes therapy. However, the possibility of better adherence to diabetes therapy cannot be overlooked. These improvements in $\mathrm{HbA}_{1} \mathrm{c}$ are consistent with the findings of a meta-analysis [1], which reviewed studies investigating the effect of exercise on $\mathrm{HbA}_{1} \mathrm{c}$ in people with Type 2 diabetes and demonstrated an overall mean difference in $\mathrm{HbA}_{1} \mathrm{c}$ of $0.66 \%$ between exercise and control conditions. The difference in $\mathrm{HbA}_{1} \mathrm{c}$ between the control and experimental groups in this study is clinically significant [1] and is close to the difference between conventional and intensive glucose-lowering therapy in the UKPDS [2]. In the UKPDS this difference was related to a significant reduction in the development and progression of diabetes complications. It should be noted that in the UKPDS this difference was maintained for a median of 10 years. Compared with intensive glucose-lowering therapy, physical activity is a potentially less expensive way of achieving this difference.

The only significant difference in lipid profile recorded in this study was a significant between-group difference in the change in total cholesterol from baseline to 12 months. A number of studies have reported changes in lipid profile after exercise in people with Type 2 diabetes [11, 8, 41, 14]. Studies demonstrating the greatest improvements tend to use moderate to high-intensity, structured exercise programmes $[11,8]$ or incorporate interventions that combine diet and physical activity $[14,15]$. Furthermore, the majority of studies have only short-term follow-up (up to 3 months). These differences in study design, in addition to the fact that mean baseline level of most lipids in the present study met current target levels [37], could explain the comparably small changes recorded.

Limited research has investigated the effect of regular physical activity on plasma fibrinogen in people with Type 2 diabetes. Results from previous research are inconsistent. Some studies report no significant changes in fibrinogen [42], while others show significant reductions [43]. In this study, a significant between-group difference in fibrinogen concentrations was recorded after 6 months. This difference was not maintained after 12 months. It is difficult to compare the results of this study to previous research. None of the previous studies in people with Type 2 diabetes include a true control group, and in the present study the significant difference recorded after 6 months is largely the result of an increase in fibrinogen in the control group.

Previous research investigating the effect of physical activity on t-PA has produced inconsistent results. Significant decreases in t-PA concentrations have been reported after 8 weeks of moderate-intensity exercise in people with Type 2 diabetes [44]. In contrast, a study investigating the short- and long-term effects of diet and energy-restrictive diet with and without exercise on t-PA in healthy, overweight, post-menopausal women [45] reported no additional benefit of physical activity. In the present study, no significant changes were recorded at any time point. Differences in exercise protocols, training status, subject health and the methods used for assessment of t-PA are probably responsible for the inconsistency in results.

Albuminuria excretion rate increases during and immediately after acute exercise, and this is associated with the rise in systolic blood pressure during physical activity and exercise [46]. There is currently no evidence suggesting that this acute effect of physical activity leads to renal impairment in the long term. In people with Type 2 diabetes, the presence of microalbuminuria is associated with increased cardiovascular disease and increased all-cause mortality [47]. In an observation study [48], higher levels of physical activity were associated with a higher prevalence of normoalbuminuria. In the present study there was no significant difference in the number of participants with evidence of microalbuminuria (defined as an albumin excretion rate of $20-300 \mathrm{mg} / 24 \mathrm{~h}$ ) at baseline, after 6 months or after 12 months.

In summary, exercise consultation was effective for promoting and maintaining physical activity. Participants receiving physical activity counselling experienced improvements in glycaemic control. These improvements are clinically significant and will substantially reduce the development and progression of diabetic complications if maintained long term. Experimental participants also recorded improvements in several cardiovascular risk factors including blood pressure, total cholesterol and fibrinogen concentrations. These improvements are of particular impor- 
tance in a population of people with Type 2 diabetes in which cardiovascular disease is the leading cause of death [49].

This study provides the evidence base for the addition of a realistic and effective physical activity intervention, which could be incorporated into current diabetes care with minimal expense. Any member of the diabetes team could be trained to conduct this intervention. We believe this study provides the support evidence that such an intervention should become part of standard diabetes care. This study suggests that without such an intervention, people with Type 2 diabetes reduce their physical activity and experience a deterioration in health.

Acknowledgements. Special thanks to all project participants. This work was supported in part by TENOVUS Scotland (30967) and by the diabetes and cardiac research funds at the Royal Alexandra Hospital, Scotland.

\section{References}

1. Boulé N, Haddad E, Kenny G, Wells G, Sigal R (2001) Effects of exercise on glycaemic control and body mass in Type 2 diabetes: A Meta-analysis of controlled clinical trials. JAMA 286:1218-1227

2. UK Prospective Diabetes Study Group (1998) Intensive blood glucose control with sulphonylureas or insulin compared with conventional treatment and risk of complications in patients with Type 2 diabetes (UKPDS). Lancet 352:837-853

3. Ruderman N, Devlin J (1995) The health professional's guide to diabetes and exercise. American Diabetes Association, Alexandria

4. Diabetes Prevention Programme Research Group (2002) Reduction in the incidence of Type 2 diabetes with lifestyle intervention or metformin. N Engl J Med 346:393-403

5. Tuomilehto J, Lindstrom J, Eriksson J et al. (2001) Prevention of Type 2 diabetes mellitus by changes in lifestyle among subjects with impaired glucose tolerance. N Engl J Med 344:1343-1350

6. Krug L, Haire-Joshu D, Heady S (1991) Exercise habits and exercise relapse in persons with non-insulin-dependent diabetes mellitus. Diabet Educ 17:185-188

7. Wilson W, Ary D, Bigard A et al. (1986) Psychosocial predictors of self-care behaviours (compliance) and glycemic control in non-insulin-dependent diabetes mellitus. Diabetes Care 9:614-622

8. Ligtenberg P, Hoekstra J, Bol E, Zonderland M, Erkelens D (1997) Effects of physical training on metabolic control in elderly type 2 diabetes mellitus patients. Clin Sci 93:127-135

9. Mourier A, Gautier J, Kerviler E et al. (1997) Mobilization of visercal adipose tissue related to the improvement in insulin sensitivity in response to physical training in NIDDM. Diabetes Care 20:385-391

10. Raz I, Hauser E, Bursztyn M (1994) Moderate exercise improves glucose metabolism in uncontrolled elderly patients with non-insulin-dependent diabetes mellitus. Isr J Med Sci 30:766-770

11. Lehmann R, Vokac A, Niedermann K, Agosti K, Spinas G (1995) Loss of abdominal fat and improvement of the cardiovascular risk profile by regular moderate exercise training in patients with NIDDM. Diabetologia 38:1313-1319
12. Lehmann R, Engler H, Honegger R, Riesen W, Spinas G (2001) Alterations of lipolytic enzymes and high-density lipoprotein subfractions induced by physical activity in type 2 diabetes mellitus. Eur J Clin Invest 31:37-44

13. Samsara K, Ashwell S, MacKintosh A et al. (1997) Will older sedentary people with non-insulin-dependent diabetes mellitus start exercising? A health promotion model. Diabetes Res Clin Pract 37:121-128

14. Vanninen E, Uusitupa M, Siitonen O, Laitinen J, Lansimies E (1992) Habitual physical activity, aerobic capacity and metabolic control in patients with newly-diagnosed Type 2 (non-insulin-dependent diabetes mellitus): effect of 1-year diet and exercise intervention. Diabetologia 35:340-346

15. Uusitupa M (1996) Early lifestyle intervention in patients with non-insulin-dependent diabetes mellitus and impaired glucose tolerance. Ann Med 28:445-449

16. Hanefeldt M, Fischer S, Schmechel H et al. (1991) Diabetes Intervention Study: multi-intervention trial in newly diagnosed NIDDM. Diabetes Care 14:308-317

17. McKay G, King D, Eakin E, Seeley J, Glasgow R (2001) The diabetes network-based physical activity intervention. Diabetes Care 24:1328-1334

18. Kirk A, Higgins L, Hughes A et al. (2001) A randomised controlled trial to study the effect of exercise consultation on the promotion of physical activity in people with Type 2 diabetes: a pilot study. Diabet Med 18:877-883

19. Hasler T, Fisher B, MacIntyre P, Mutrie N (2000) Exercise consultation and physical activity in patients with Type 1 diabetes. Prac Diab Int 17:44-48

20. Smith D, Heckemeyer C, Kratt P, Manson D (1997) Motivational interviewing to improve adherence to a behavioural weight-control programme for older obese women with NIDDM. Diabetes Care 20:52-54

21. Di Loreto C, Fanelli P, Lucidi P et al. (2003) Validation of a counselling strategy to promote the adoption and the maintenance of physical activity by Type 2 diabetes subjects. Diabetes Care 26:404-408

22. Kahn E, Ramsey L, Brownson R et al. (2002) The effectiveness of interventions to increase physical activity. Am J Prev Med 22:73-107

23. Lowther M, Mutrie N, Scott E (2002) Promoting physical activity in a socially and economically deprived community: a 12 month randomised control trial of fitness assessment and exercise consultation. J Sports Sci 20:577-588

24. Proshaska J, Marcus B (1994) The transtheoretical model: application to exercise. In: Dishman R (ed) Advances in exercise adherence. Human Kinetics, Georgia, pp 161-180

25. Loughlan C, Mutrie N (1995) Conducting exercise consultation guidelines for health professionals. J Inst Health Educ 33:78-82

26. Kirk A, Mutrie N, MacIntyre P, Fisher M (2003) Increasing physical activity in people with Type 2 diabetes. Diabetes Care 26:1186-1192

27. The Expert Committee on the Diagnosis and Classification of Diabetes Mellitus (2000) Report of the Expert Committee on the Diagnosis and Classification of Diabetes Mellitus. Diabetes Care 23:S4-S20

28. Melanson E, Freedson P (1995) Validity of the Computer Science and Applications, Inc (CSA) activity monitor. Med Sci Sports Exerc 27:934-940

29. Sallis JF, Haskell WL, Wood PD et al. (1985) Physical activity assessment methodology in the Five City Project. Am J Epidemiol 121:91-106

30. Pate R, Pratt M, Blair S et al. (1995) Physical activity and public health: a recommendation from the Centers for Disease Control and prevention and the American College of Sports Medicine. JAMA 273:402-407 
31. American College of Sports Medicine (1990) Position stand on the recommended quality and quantity of exercise for developing and maintaining cardiorespiratory and muscular fitness in healthy adults. Med Sci Sports Exerc 22:265-274

32. Bodor G (1992) Standardisation of glycohaemoglobin determination in the clinical laboratory: three years of experience. Clin Chem 38:2414-2418

33. Siedel J, Hagele E, Ziegenhorn J, Wahlefeld A (1983) Reagent for the enzymatic determination of serum total cholesterol with improved lipolytic efficiency. Clin Chem 29:1075-1080

34. Friedewald N, Nevy R, Frederickson D (1972) Estimation of the concentration of LDL cholesterol in plasma without the use of preparative ultracentrifuge. Clin Chem 18:499-502

35. Clauss A (1957) Rapid physiological coagulation method for the determination of fibrinogen. Acta Haematol 17:237

36. Ranby M, Bergsdorf N, Nilsson T et al. (1986) Age dependence of tissue plasminogen activator concentrations in plasma, as studied by an improved enzyme-linked immunosorbent assay. Clin Chem 32:2160-2165

37. Scottish Intercollegiate Guidelines Network (SIGN) (1997) Management of diabetic cardiovascular disease. SIGN publication no. 55, Edinburgh, Scotland

38. Fagard R (2001) Exercise characteristics and the blood pressure response to dynamic physical activity. Med Sci Sports Exerc 33:S484-S492

39. Whelton S, Chin A, Xin X, He J (2002) Effect of aerobic exercise on blood pressure: a meta-analysis of randomised, controlled trials. Ann Intern Med 136:493-503

40. UK Prospective Diabetes Study Group (2002) Tight blood pressure control and the risk of macrovascular and microvascular complications in type 2 diabetes UKPDS 38. BMJ 317:703-713
41. Walker K, Piers L, Putt R, Jones J, O’Dea K (1999) Effects of regular walking on cardiovascular risk factors and body composition in normoglycemic women and women with type 2 diabetes. Diabetes Care 22:555-561

42. Schneider S, Kim H, Khachadurian A, Ruderman N (1998) Impaired fibrinolytic response to exercise in Type II diabetes: effects of exercise and physical training. Metabolism 37:924-929

43. Hornsby W, Boggess K, Lyons T et al. (1990) Hemostatic alterations with exercise conditioning in NIIDM. Diabetes Care 13:87-92

44. Dunstan D, Puddey I, Burke V et al. (1998) Exercise and fish intake: effects on serum lipids and glycaemic control for type 2 diabetics. Diabetes 15:34-37

45. Svendsen O, Hassager C, Christiansen C, Nielsen J, Winther K (1995) Plasminogen activator inhibitor-1, tissue-type plasminogen activator, and fibrinogen: effect of dieting with and without exercise in overweight postmenopausal women. Arterioscler Thromb Vasc Biol 16:381-385

46. Dahlquist G, Aperia A, Carlsson L et al. (1983) Effect of metabolic control and duration on exercise induced albuminuria in diabetic teenagers. Acta Paediatr Scand 72:895-902

47. Valmadrid C, Klein R, Moss S, Klein B (2000) The risk of cardiovascular disease mortality associated with microalbuminuria and gross proteinuria in persons with older onset diabetes mellitus. Arch Intern Med 160:1093-1100

48. Calle-Pascual A, Martin-Alvarez P, Reyes C, Calle J (2001) Regular physical activity and reduced occurrence of microalbuminuria in Type 2 diabetic patients. Diabetes Metab 19:304-309

49. Haffner S, Lehto S, Ronnemaa T, Pyorala K, Laakso M (1998) Mortality from coronary heart disease in subjects with Type 2 diabetes and in nondiabetic subjects with and without prior myocardial infarction. $\mathrm{N}$ Engl $\mathrm{J}$ Med 339:229-234 